\title{
La ventanilla única de Comercio Exterior como medida de facilitación al Comercio Exterior
}

Marco Antonio Huamán Sialer

Vocal Presidente de la Sala de Aduanas (Sala 6) del Tribunal Fiscal del Perú. Profesor en la Universidad Nacional Mayor de San Marcos, Pontificia Universidad Católica del Perú, Universidad de ESAN y Universidad Alas Peruanas. Profesor Visitante en la Universidad Nacional de Trujillo, Universidad Antenor Orrego de Trujillo, Universidad Particular de Chiclayo, Universidad Nacional Pedro Ruiz Gallo de Lambayeque y Universidad Católica San Pablo de Arequipa.

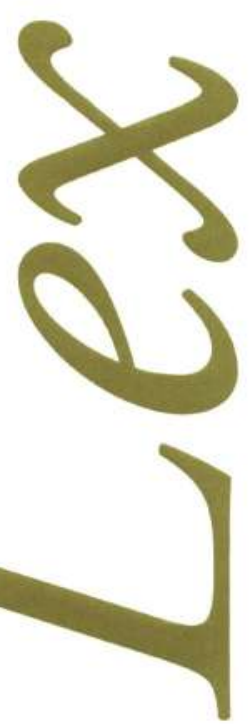


abemos que el comercio globalizado se caracteriza por su dinamismo y cambio, al punto que la operatividad de las actividades económicas subyacentes tiende a variar. En ese sentido, es necesario que los Estados nacionales -en lo que a materia aduanera importa- estén siempre atentos a cualquier cambio que se pueda producir en la operatividad de estas actividades económicas, para responder con eficacia y eficiencia a dichos cambios, y así no entorpecer su misma agilidad.

Nuestro país, con el transcurrir de los años, ha venido insertándose de a poco en este mundo real del intercambio internacional. En esa perspectiva debe destacarse la Ley N ${ }^{\circ} 28977$ (Ley de Facilitación del Comercio Exterior, y en adelante «la LFCE»), publicada en el diario oficial $E l$ Peruano el 8 de febrero de $2007^{1}$, que de alguna manera sienta las bases para lograr estándares internacionales en lo que respecta a procedimientos aduaneros modernos, que permitan a su vez reducir los plazos de demora en los trámites aduaneros y, por ende, también aminorar los sobrecostos que hoy por hoy soportan los operadores de Comercio Exterior.

Con la LFCE se busca atender dos aristas muy importantes para el desarrollo de las actividades económicas del país:

a) El avance en la liberación del comercio exterior, con el fin de incentivarla, simplificando para ello los trámites de la operatividad aduanera y promoviendo su descentralización.

La vigencia de dicha norma fue establecida de forma progresiva, conforme lo indica su Tercera Disposición Complementaria y Final. 
b) La implementación de las medidas necesarias para el cumplimiento de los compromisos relativos a los Procedimientos Aduaneros y Facilitación del Comercio comprendidos en los Acuerdos Comerciales suscritos por el Perú.

Es necesario señalar que estas medidas fueron recogidas en el Acuerdo de Promoción Comercial Perú-Estados Unidos (APC), principal motivo para la promulgación de la LFCE.

De esta manera, el Perú no solo cumplirá indefectiblemente los compromisos asumidos frente al APC, sino se adecuará a las exigencias del comercio internacional referidas a la celeridad en el control aduanero y en el despacho de las mercancías que ingresan o salen de nuestro territorio.

Bajo este escenario, la LFCE contiene diversas disposiciones que buscan promover la simplicidad y agilidad en el tratamiento aduanero del comercio exterior, a fin de de situarnos a la par con aquellas naciones del mundo más avanzadas en materia de liberación del comercio.

En cuanto a las adecuaciones a los acuerdos comerciales, la misma LFCE ha considerado la necesidad de abordar:

a) La transparencia y difusión de las normas, básicamente a cargo de la Administración Aduanera.

b) El despacho aduanero en 48 horas, conforme al artículo $35^{\circ}$ de la Ley General de Aduanas (LGA).

c) La evaluación del nivel de riesgo, debiendo la Administración Aduanera implementar sistemas electrónicos automatizados elaborados sobre la base de datos suministrados por los operadores de comercio exterior.

d) La creación del Comité de Cooperación Aduanera Interinstitucional a cargo del Ministerio de Comercio Exterior y Turismo (MINCETUR), en coordinación con el Ministerio de Economía y Finanzas (MEF).

e) Los envíos de entrega rápida, garantizando un servicio eficiente e inmediato, sin perjuicio del control aduanero posterior.

f) Las resoluciones anticipadas relacionadas con la clasificación arancelaria y valoración aduanera de las mercancías, y sobre la aplicación de devoluciones, suspensiones y exoneraciones de aranceles aduaneros y la reimportación de mercancías reparadas o alteradas en el territorio de un país que ha suscrito acuerdos comerciales con el Perú.

Ahora bien, entre las medidas de facilitación al comercio exterior se consideraron:

a) La implementación de la Ventanilla Única de Comercio Exterior a cargo del MINCETUR.

b) La publicación del contenido y precios de los servicios portuarios y aeroportuarios a cargo del Ministerio de Transportes y Comunicaciones.

c) La promoción de los puertos interiores del país, conforme al artículo $56^{\circ}$ de la LGA. 
d) La ampliación del plazo del régimen de depósito, de acuerdo al segundo párrafo del artículo 60 de la LGA.

e) La precisión de que las mercancías nacionales o nacionalizadas sometidas a los regímenes de exportación definitiva o temporal no constituyen mercancías susceptibles de la aplicación de la sanción prevista en el inciso i) del artículo $108^{\circ}$ de la LGA por no afectar el interés fiscal.

f) Las sanciones en los envíos de entrega rápida, conforme al artículo $109^{\circ}$ de la LGA.

Por su especial relevancia, en esta oportunidad, nos abocaremos a la medida de facilitación al comercio exterior denominada Ventanilla Única de Comercio Exterior (en adelante VUCE).

\section{Antecedentes Internacionales de la VUCE}

Uno de los objetivos de las Naciones Unidas es promover el comercio por su potencial para fomentar el desarrollo económico y erradicar la pobreza. Así, el Centro de las Naciones Unidas para la Facilitación del Comercio y el Comercio Electrónico (UN/CEFACT por sus siglas en inglés), tiene la misión de mejorar la habilidad de los negocios, comercio y organizaciones administrativas, tanto de economías desarrolladas como de economías en desarrollo y en transición, para intercambiar productos y servicios relevantes efectivamente.

Su principal objetivo es facilitar las transacciones nacionales e internacionales, a través de la simplificación y la armonización de procesos, procedimientos y flujos de información, y, de esta forma, contribuir al crecimiento del comercio global.

En tal sentido, el UN/CEFACT ha venido emitiendo una serie de recomendaciones precisamente dirigidas a la facilitación del comercio exterior. Bajo este contexto, en julio de 2005 emitió la Recomendación No $33^{2}$ - Establishing a Single Window ${ }^{3}$, para mejorar el intercambio eficiente de información sobre el comercio exterior entre los sujetos involucrados en este tipo de operaciones.

Dicha recomendación fue aprobada en septiembre de 2004 por un grupo de trabajo por encargo del UN/CEFACT.

El documento puede verse en: http://www.unece.org/cefact/recommendations/rec33/rec33_trd352e.pdf 


\section{Cuadro $\mathrm{N}^{\circ} 1$ \\ DEFINICIÓN DE LA VUCE Según el UN/CEFACT}

La Ventanilla Única (Single Window) es el mecanismo de facilitación que permite a las partes que se encuentran involucradas en el comercio y el transporte presentar información y documentos estandarizados en un solo punto de entrada para cumplir con todos los trámites de importación, exportación y tránsito. En caso la información fuese electrónica, la misma debe ser remitida una sola vez.

La Recomendación № 33 parte de la premisa de que en muchos países las compañías involucradas en el comercio internacional deben regularmente preparar y enviar largos volúmenes de información y de documentos a las autoridades gubernamentales para acceder a los requerimientos legales de la importación, exportación y aquellos relacionados con el tránsito. Además, esta información y documentación usualmente deben ser enviadas a través de numerosas agencias u oficinas, cada una de ellas con sus formularios y sistemas específicos (manual o automatizado). Estos extensos requisitos, junto con los costos de procedimientos asociados a ellos, pueden constituir una seria carga para los gobiernos y para la comunidad comercial, y pueden también ser una seria barrera al desarrollo del comercio internacional y el desarrollo en general.

En esa medida, un acercamiento para dirigir este problema es el establecimiento de una VUCE, mediante la cual la información relacionada con el comercio y/o los documentos necesarios solo necesitan ser enviados una vez en un solo punto de entrada, a efectos de mejorar la disponibilidad y el manejo de la información, dinamizar y simplificar el flujo de información entre los comerciantes y el Gobierno. Entonces, para el UN/CEFACT la VUCE supone poner en práctica los conceptos de facilitación del comercio exterior.

La VUCE, en general, es manejada centralizadamente, permitiendo a las agencias y autoridades gubernamentales recibir o tener acceso a la información relevante para sus fines. Adicionalmente, las autoridades participantes y las agencias deben coordinar sus controles. En algunos casos, la VUCE puede proveer de facilidades para el pago de determinados tributos.

Por tal motivo, la Recomendación № 33 invoca a que:

1. Se considere la posibilidad de implementar una VUCE en cada país. 
2. Se establezca una VUCE a nivel nacional a través de un esfuerzo colaborativo con todas las autoridades gubernamentales y la comunidad empresarial.

3. Se tomen en consideración las Directrices Anexas para el establecimiento de una VUCE 4 .

\section{Antecedentes Nacionales de la VUCE}

La importancia del comercio exterior en nuestro país motivó que en el marco del Plan Nacional Exportador (PENX 2003-2013) y el Plan Nacional de Competitividad (D. S. No 057 2005-PCM) se planteara la simplificación de trámites para este tipo de operaciones.

En el año 2003, la SUNAT ya venía trabajando un Proyecto de Interconexión entre entidades públicas dirigido a la integración de procesos para el despacho de mercancías.

A fines del 2005, a la labor de la Superintendencia Nacional de Administración Tributaria (SUNAT) se sumó el MINCETUR, con el fin de trabajar conjuntamente en la elaboración de un proyecto mayor, elaborando el plan de desarrollo de lo que sería la VUCE, tomando en cuenta algunos estudios para su implementación en nuestro país.

Entre abril y mayo del 2006, MINCETUR y SUNAT concluyeron las presentaciones de la propuesta de la VUCE ante el Consejo Nacional de Competitividad (CNC) de la Presidencia del Consejo de Ministros, consiguiéndose su aprobación.

\section{Principales problemas en el Comercio Exterior Peruano}

En nuestro país se vienen presentando principalmente los siguientes inconvenientes en el comercio exterior:

1. Los trámites no son virtuales.

2. El incumplimiento de plazos y costos proyectados.

3. La duplicidad de requerimientos por parte de entidades públicas.

4. La existencia de 15 instituciones gubernamentales vinculadas al comercio de mercancías restringidas.

5. La inexistencia de integración entre las entidades de control.

6. La falta de coordinación entre los órganos encargados del control paraaduanero de las mercancías con la Aduana sobre el momento de la verificación física.

\footnotetext{
4 Estas Directrices forman la Segunda Parte de la Recomendación N ${ }^{\circ} 33$, a la cual sirven de complemento. Ellas proveen una descripción de las cuestiones principales que deben ser atendidas, algunas de las herramientas disponibles y los pasos a seguir para establecer una VUCE.
} 
Una excelente medida, que nos coloca a la par con los países más avanzados en temas de integración comercial, es la creación de la VUCE, pues a través de ella se busca integrar y simplificar los procesos y servicios de las instituciones del Estado vinculadas con el comercio exterior, asegurando el cumplimiento y el control eficiente de las operaciones.

De esta manera se logrará integrar los trámites aduaneros para hacerlos más simples y ágiles, generando estabilidad jurídica en los operadores de comercio exterior, lo que es plausible desde el punto de vista del ahorro en tiempo, dinero y predictibilidad en los trámites iniciados, ya que además se obtendrá la revisión física simultánea de las mercancías y un control integrado por las instituciones intervinientes que permitan a su vez ejercer una efectiva gestión de riesgo.

Asimismo, si todo redunda en un trámite ágil y de control eficiente de las operaciones, resulta lógico que se dé validez a los documentos electrónicos generados en los trámites de la VUCE, siendo además pertinente que el Estado no requiera por ningún motivo aquella información electrónica del administrado de la que ya disponga.

\section{La creación de la VUCE en el Perú}

La VUCE fue creada por el artículo $1^{\circ}$ del D. S. No 165-2006-EF, publicado el 3 de noviembre de 2006, en atención al ritmo acelerado del crecimiento del comercio exterior, que exigía asegurar su sostenibilidad futura, transparencia y rapidez en los trámites, con la finalidad de reducir la diversidad y dispersión de trámites, brindar una respuesta oportuna y rápida ante los requerimientos de los importadores, exportadores y de los propios mercados internacionales, así como facilitar en términos de menos costos y plazos las operaciones de importación y exportación en general5.

La misma norma dispuso la implementación de la VUCE con el uso de medios electrónicos para la obtención de permisos, certificaciones, licencias y demás autorizaciones que se exigen ante las entidades del Estado para la realización de las operaciones de importación y exportación de mercancías, estableciendo además las pautas necesarias para su ejecución, entre ellas la crea-

\footnotetext{
5 Tal como aparece en el primer considerando del citado D. S. No 165-2006-EF. En el segundo considerando de la misma norma se señaló que a fin de lograr el objetivo planteado, que conlleve a una mayor facilitación del comercio exterior, era necesario sistematizar e integrar los procedimientos y trámites administrativos, para en una segunda etapa simplificar y uniformizar su documentación a través del diseño e implementación de un sistema de ventanilla única para los trámites que requieren realizar los exportadores e importadores del país ante las distintas entidades del Estado, teniendo como base el acceso y la gestión compartida por todos los agentes participantes en las operaciones de comercio exterior, a través de la coordinación e integración de las entidades del Estado y del sector privado que intervienen en el procesamiento de la información.
} 
ción de una Comisión Multisectorial encargada de implementar y poner en funcionamiento dicha medida de facilitación al comercio exterior.

El mencionado decreto fue modificado por el D. S. No 199-2006-EF, publicado el 14 de diciembre de 2006, para establecer que la administración de la VUCE debía estar a cargo del MINCETUR y ya no de la SUNAT ${ }^{6}$, y que los documentos electrónicos o digitalizados utilizados en la VUCE tendrían la misma validez que el documento original, debiendo ser aceptados como tal por las entidades del Estado, de conformidad con lo establecido en la Ley del Procedimiento Administrativo General.

\section{La dación de la ley de facilitación de Comercio Exterior y la VUCE}

Ahora bien, como ya se adelantó, con la dación de la LFCE se dictan nuevas precisiones referidas al funcionamiento de la VUCE, y en forma concordante con el D. S. No 199-2006EF, el artículo $9^{\circ}$ de la citada Ley dispuso que esta estará a cargo del MINCETUR y permitirá a los operadores de comercio exterior tramitar las autorizaciones y permisos que exigen las entidades competentes para importar o exportar mercancías.

Dicha LFCE dispone que el MINCETUR designará una Comisión Especial, en coordinación con las demás entidades competentes, para la uniformización y simplificación de los trámites que se efectuarán en dicha ventanilla, la que incluirá la tramitación por medios electrónicos. Y dispone también que el MINCETUR deberá expedir el documento autorizante requerido para el cumplimiento de los trámites de importación y exportación en el plazo de 5 días computados a partir de la fecha de presentación de la solicitud, la que, inclusive, puede ser presentada por medios electrónicos conjuntamente con la documentación requerida por dicha institución. Vencido el plazo, se aplicará el silencio administrativo positivo, es decir, se considerará aprobada la solicitud presentada por el interesado.

Finalmente, la Primera Disposición Complementaria y Final de la LFCE facultan al MINCETUR para que, en un plazo no mayor de 90 días, dicte las disposiciones reglamentarias para la implementación de la VUCE. Y es justamente en virtud de dicho mandato que el Ministerio aprobó el D. S. No 10-2007-MINCETUR.

6 Asimismo, se indicó que la administración del MINCETUR está referida a la gestión, control, coordinación y solución de conflictos en la operatividad de la VUCE. 


\section{La reglamentación de la VUCE}

El artículo $1^{\circ}$ del D. S. No 10-2007-MINCETUR, publicado el 28 de octubre de 2007, aprobó el Reglamento para la implementación de la Ventanilla Única de Comercio Exterior VUCE (en adelante "el Reglamento"), dejando sin efecto, acertadamente, las disposiciones contenidas en el D. S. No 165-2006-EF, con excepción del artículo $1^{\circ}$, pues existiendo nuevos parámetros propuestos por la LFCE, era necesario ceñirse al camino trazado por la referida Ley.

Es importante resaltar que el Reglamento ha precisado que es de cumplimiento obligatorio para los operadores de comercio exterior y las entidades de la administración pública integrantes y/o vinculadas a la VUCE.

Entre otras disposiciones ${ }^{7}$, en el artículo $16^{\circ}$ de esta norma se establece que el plazo de 5 días para resolver las solicitudes de los administrados, establecido en el artículo $9^{\circ}$ de la Ley LFCE, se computará desde la presentación de la solicitud por parte del administrado, cumpliendo con todos los requisitos y documentos exigidos por la normativa vigente, entre los cuales se incluye la puesta a disposición de la mercancía cuando se requiera inspección física de la misma, el resultado de los análisis y/o pruebas de laboratorio, y la asistencia de terceros a las diligencias fuera de la sede de la entidad.

Una vez cumplidas tales exigencias, se notificará al administrado la fecha en que se inicia el cómputo del plazo para resolver; vencido el mismo sin haberse resuelto, la solicitud se considerará aprobada.

Por lo visto hasta aquí, consideramos que la creación y reglamentación de la VUCE constituyen un avance importante en el establecimiento de un instrumento vital para agilizar los trámites que los importadores y exportadores deben gestionar ante los diversos estamentos de la Administración Pública, tales como certificaciones, permisos, autorizaciones, licencias, entre otros requeridos para el ingreso o salida de mercancías, pues la demora que significa obtenerlos puede encontrar una solución viable con la tramitación en una plataforma única por vía electrónica, lo que permitirá interconectar al interesado tanto con la Administración Aduanera como con las demás instituciones públicas.

\footnotetext{
${ }_{7}$ Pueden leerse mayores referencias sobre el Reglamento en «Ventanilla Única de Comercio Exterior: Disposiciones reglamentarias" (Decreto Supremo N 010-2007-MINCETUR). En: Revista Análisis Tributario, N 238, noviembre de 2007, pp. 14 y 15 .
} 


\title{
Cuadro $\mathrm{N}^{\circ} 2$
}

\section{OBJETIVOS DE LA VUCE}

\author{
Según D. S. No 10-2007-MINCETUR
}

Son objetivos de la VUCE:

- Simplificar y uniformizar los procedimientos, formularios y plazos de los trámites que se efectúan ante las entidades de la Administración Pública para la obtención de los permisos, certificaciones, licencias y demás autorizaciones para la realización de operaciones de importación o exportación de mercancías.

- Canalizar la gestión de los trámites que se efectúan ante las entidades de la Administración Pública para las operaciones de importación y exportación de mercancías, a través de sistemas electrónicos que faciliten el acceso de todos los operadores de comercio exterior.

- Permitir el intercambio de información de manera electrónica entre las entidades de la Administración Pública integrantes de la VUCE y entre estas y las demás entidades de la administración pública que, no integrando la VUCE, cuenten con la información necesaria para efectuar los trámites referidos a operaciones de importación o exportación de mercancías.

\section{Principales avances sobre la VUCE}

El Consejo Nacional de Usuarios del Sistema de Distribución Física Internacional de Mercancías (CONUDFI) ${ }^{8}$ y la empresa Porte Servicios Telemáticos de España ganaron la licitación realizada por el MINCETUR para diseñar e implementar la VUCE en el Perú.

Hoy en día se ha previsto el desarrollo de la fase inicial de la VUCE en dos etapas:

a) La interconexión física, que será realizada por la SUNAT con recursos propios y del Banco Interamericano de Desarrollo.

b) La implementación y puesta en marcha de la VUCE, que será financiada por la CAF y la UE.

Para la interconexión física, se han seleccionado los siguientes procedimientos:

8 El CONUDFI es una asociación civil sin fines de lucro, integrada por la Asociación de Exportadores (ADEX), la Cámara de Comercio de Lima (CCL), la Sociedad Nacional de Industrias (SNI), la Cámara Nacional de Comercio Producción y Servicios (Perú Cámaras), la Asociación de Agentes de Aduanas del Perú (AAAP) y la Asociación Civil Frío Aéreo. 
SENASA : Importación Sanidad Vegetal.

DIGEMID : Importación de psicotrópicos y precursores.

DIGESA : Importación de alimentos y bebidas.

PRODUCE : Importación de químicos y productos fiscalizados.

MTC : Importación de equipos y aparatos de telecomunicación.

Cabe resaltar que no todas las entidades vinculadas a este proceso tienen la misma capacidad de software y equipamiento, lo cual de alguna manera ha demorado el proceso en marcha; pero, a pesar de ello, esperamos que siga en camino el objetivo propuesto, debiendo manifestarse que hoy en día se está diseñando la página web de la VUCE, lo que indica que el sistema aún no es operativo.

Esta interconexión tiene por objetivo que las instituciones del Estado puedan conversar entre sí, intercambiar información y poder optimizar algunos procedimientos, aunque lo más conveniente resulte ser la optimización de la ventanilla propiamente dicha.

Como sabemos, actualmente cada institución pública que interviene en el comercio exterior tiene sus propios procedimientos y trámites, los cuales son manejados independientemente. Ahora, lo que se busca con la VUCE es el trabajo de todos en forma conjunta, a fin de obtener como resultado la disminución del tiempo que toman tales procedimientos y trámites.

Según la opinión de quienes tienen vinculación con el tema, la VUCE estaría concluida para julio de 2008.

\section{A manera de conclusiones}

- La VUCE se crea para simplificar y optimizar los procedimientos; dicho de otro modo, para que los operadores de comercio exterior puedan ver mejorada su competitividad, reducir sus costos a través de la reducción de tiempos en los procedimientos y trámites, para una ágil declaración y levante, así como la aplicación de reglas predecibles.

- Desde el punto de vista de las ventajas para el Estado, se conseguirá mayor efectividad y eficiencia en la utilización de los recursos, una mejor gestión de riesgo aduanero, una mayor transparencia y un incremento en la seguridad.

- De esta manera será posible contar con un sistema integrado de control de la infraestructura de comercio exterior, sobre la base de comunicaciones interinstitucionales y los TUPA de comercio exterior armonizado. 